\title{
THE GALACTIC BULGE SURVEY: OUTLINE AND X-RAY OBSERVATIONS
}

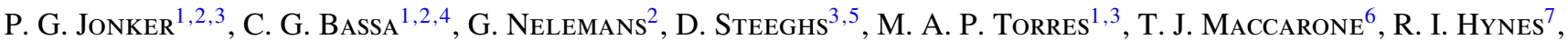 \\ S. Greiss ${ }^{5}$, J. Clem ${ }^{7}$, A. Dieball ${ }^{6}$, V. J. Mikles ${ }^{7}$, C. T. Britt ${ }^{7}$, L. Gossen ${ }^{7}$, A. C. Collazzi ${ }^{7}$, R. WiJnands ${ }^{8}$, J. J. M. In' $^{\prime}$ \\ ZAnd $^{1}$, M. Méndez ${ }^{9}$, N. ReA ${ }^{10}$, E. Kunlkers ${ }^{11}$, E. M. Ratti ${ }^{1}$, L. M. van HaAften ${ }^{2}$, C. Heinke ${ }^{12}$, F. Özel ${ }^{13}$, P. J. Groot ${ }^{2}$, \\ AND F. VERBUNT ${ }^{1,14}$ \\ ${ }^{1}$ SRON, Netherlands Institute for Space Research, Sorbonnelaan 2, 3584 CA, Utrecht, The Netherlands; p.jonker@sron.nl \\ ${ }^{2}$ Department of Astrophysics, IMAPP, Radboud University Nijmegen, Heyendaalseweg 135, 6525 AJ, Nijmegen, The Netherlands \\ ${ }^{3}$ Harvard-Smithsonian Center for Astrophysics, 60 Garden Street, Cambridge, MA 02138, USA \\ ${ }^{4}$ Jodrell Bank Centre for Astrophysics, School of Physics and Astronomy, University of Manchester, Manchester M13 9PL, UK \\ ${ }^{5}$ Astronomy and Astrophysics, Department of Physics, University of Warwick, Coventry, CV4 7AL, UK \\ ${ }^{6}$ School of Physics and Astronomy, University of Southampton, Southampton SO17 1BJ, UK \\ ${ }^{7}$ Department of Physics and Astronomy, Louisiana State University, Baton Rouge, LA 70803-4001, USA \\ ${ }^{8}$ Astronomical Institute "Anton Pannekoek," University of Amsterdam, Kruislaan 403, 1098 SJ Amsterdam, The Netherlands \\ ${ }^{9}$ Kapteyn Astronomical Institute, University of Groningen, P.O. Box 800, 9700 AV Groningen, The Netherlands \\ ${ }^{10}$ Institut de Ciencies de l'Espai (ICE, IEEC-CSIC), Campus UAB, Fac. de Ciencies, Torre C5-parell, 2a planta, 08193, Barcelona, Spain \\ ${ }^{11}$ ISOC, ESA/ESAC, Urb. Villafranca del Castillo, P.O. Box 50727, 28080 Madrid, Spain \\ 12 Department of Physics, University of Alberta, Room 238 CEB, Edmonton, AB T6G 2G7, Canada \\ ${ }^{13}$ Steward Observatory, University of Arizona, Tucson, AZ 85721, USA \\ ${ }^{14}$ Astronomical Institute, Utrecht University, P.O. Box 80000, 3508 TA, Utrecht, The Netherlands \\ Received 2011 January 14; accepted 2011 March 4; published 2011 April 29
}

\begin{abstract}
We introduce the Galactic Bulge Survey (GBS) and we provide the Chandra source list for the region that has been observed to date. Among the goals of the GBS are constraining the neutron star (NS) equation of state and the black hole $(\mathrm{BH})$ mass distribution via the identification of eclipsing NS and BH low-mass X-ray binaries (LMXBs). The latter goal will, in addition, be obtained by significantly enlarging the number of BH systems for which a BH mass can be derived. Further goals include constraining X-ray binary formation scenarios, in particular the common envelope phase and the occurrence of kicks, via source-type number counts and an investigation of the spatial distribution of X-ray binaries, respectively. The GBS targets two strips of $6^{\circ} \times 1^{\circ}\left(12 \mathrm{deg}^{2}\right.$ in total), one above $\left(1^{\circ}<b<2^{\circ}\right)$ and one below $\left(-2^{\circ}<b<-1^{\circ}\right)$ the Galactic plane in the direction of the Galactic center at both X-ray and optical wavelengths. By avoiding the Galactic plane $\left(-1^{\circ}<b<1^{\circ}\right)$ we limit the influence of extinction on the X-ray and optical emission but still sample relatively large number densities of sources. The survey is designed such that a large fraction of the X-ray sources can be identified from their optical spectra. The $\mathrm{X}$-ray survey, by design, covers a large area on the sky while the depth is shallow using 2 ks per Chandra pointing. In this way we maximize the predicted number ratio of (quiescent) LMXBs to cataclysmic variables. The survey is approximately homogeneous in depth to a $0.5-10 \mathrm{keV}$ flux of $7.7 \times 10^{-14} \mathrm{erg} \mathrm{cm}^{-2} \mathrm{~s}^{-1}$. So far, we have covered about two-thirds $\left(8.3 \mathrm{deg}^{2}\right)$ of the projected survey area with Chandra providing over 1200 unique X-ray sources. We discuss the characteristics and the variability of the brightest of these sources.
\end{abstract}

Key words: accretion, accretion disks - binaries: close - X-rays: binaries

Online-only material: color figures, machine-readable table

\section{INTRODUCTION}

\subsection{Multi-wavelength Observations of X-ray Sources}

$\mathrm{X}$-ray observations are excellent probes of coronally active and accreting sources. Whereas studies of X-ray sources in our Galaxy have to a large extent focussed on bright systems, investigations of fainter source classes have typically been done in the Galactic center (e.g., Muno et al. 2003). There, however, crowding and extinction make it more difficult to identify the correct optical and/or infrared counterparts for large fractions of the sources (e.g., Mauerhan et al. 2009).

It is clear from previous surveys that multi-wavelength observations are vital for classifying faint X-ray sources since $\mathrm{X}$-ray spectral information alone is rarely sufficient. Classification is important for various science goals as we outline below.

The Galactic Bulge Survey (GBS) we present in this paper was designed to allow multi-wavelength observations of the detected X-ray sources. The GBS consists of Chandra and optical imaging of two strips of $6^{\circ} \times 1^{\circ}$, one centered 1.5 above the Galactic plane and the other 1.5 below the plane. We have chosen this area as the source density is still high, but by excluding $|b|<1^{\circ}$ we avoid the regions that are most heavily affected by extinction and source confusion (see Figure 1). In Table 1 we provide a reference table for the acronyms used in this paper.

\subsection{Goals of the GBS: Compact Object Masses}

The discovery of a large sample of X-ray sources will allow us to identify rare $\mathrm{X}$-ray binaries such as quiescent eclipsing black hole (BH) and neutron star (NS) low-mass X-ray binaries (LMXBs) and ultra-compact X-ray binaries (UCXBs; LMXBs that have orbital periods $<1 \mathrm{hr}$ ). For dynamical mass measurements in LMXBs one needs to measure three parameters: the radial velocity amplitude of the companion star $\left(K_{2}\right)$, the ratio between the mass of the companion star and the NS or BH mass $(q)$, and the inclination $(i)$. A measurement of the rotational 


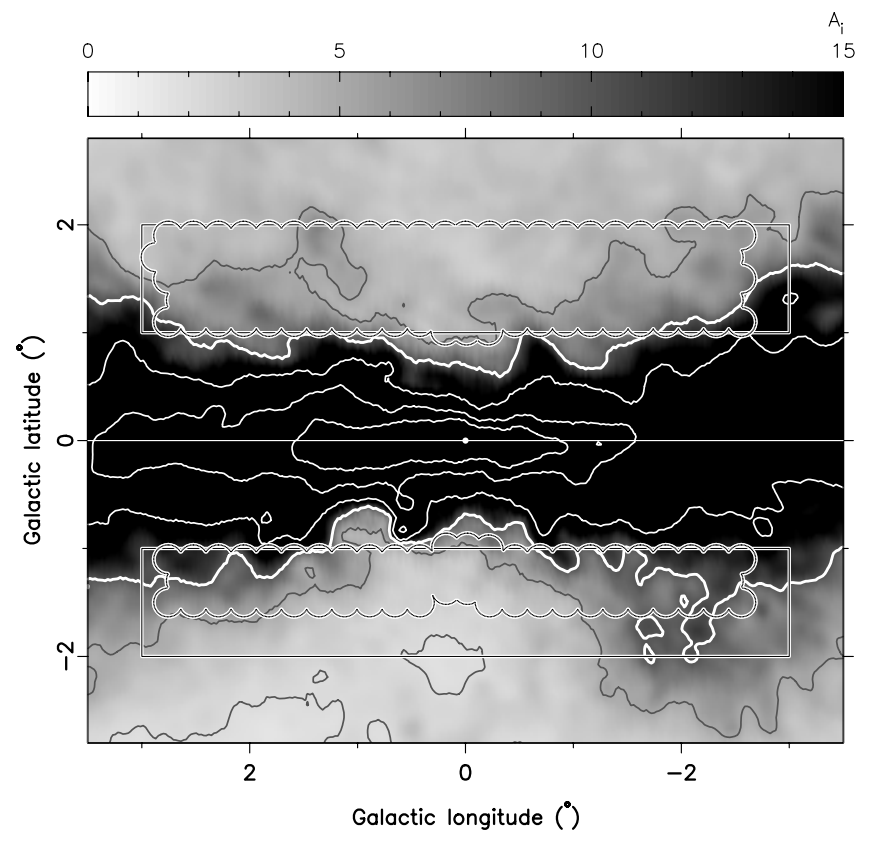

Figure 1. Large black plus white rimmed boxes indicate the two $6^{\circ} \times 1^{\circ} \mathrm{GBS}$ fields in Galactic coordinates. The thick white contour made-up of small circles each of a $14^{\prime}$ diameter indicates the area covered by our Chandra observations until 2010. The gray-scale image and contours depict the total absorption in the Sloan $i^{\prime}$-band filter, $A_{i^{\prime}}$, estimated from the CoBE dust maps (Schlegel et al. 1998). The contours are at $A_{i^{\prime}}$ values of 2,5 (both in dark gray), 10, 20, 50, and 100 (in white).

broadening of the stellar absorption lines $(v \sin i)$ combined with $K_{2}$ gives this determination of $q$. The reason is that in Roche lobe overflow systems, like LMXBs, the companion star is tidally forced to co-rotate with the binary orbit (Tassoul 1988).

The system inclination can be determined through modeling of ellipsoidal variations caused by distortion of the companion star using multi-color optical light curves (e.g., Orosz \& Hauschildt 2000, Cantrell et al. 2010). In systems with favorable viewing angles, the (X-ray) eclipse duration can be used to accurately determine the inclination (Horne 1985). Since the inclination is constrained by the geometry, mass measurements in eclipsing systems are independent of the modeling that lies behind inclinations derived from ellipsoidal variations. Quiescent eclipsing systems are prime targets for optical mass measurements. Such mass measurements provide constraints on the NS equation of state (EoS) and on the dividing line between NS and BH systems (e.g., Özel et al. 2010). Constraining the NS EoS remains one of the ultimate goals for NS studies (Lattimer \& Prakash 2004 and references therein) and mass measurements of BH systems would improve our estimates of the stellar mass BH mass distribution with implications for supernova and Gamma-ray burst modeling. Furthermore, the BH sample selected in quiescence, as in the GBS, is not susceptible to potential X-ray outburst duty cycle based selection effects that occur when selecting BHs after they have become X-ray bright and returned to quiescence, which is now common practice. Finding eclipsing LMXBs with optical or near-infrared counterparts bright enough that optical or near-infrared spectroscopy can provide accurate mass measurements is the principal goal of the GBS.

\subsection{Goals of the GBS: Binary Formation and Evolution}

The second main goal of the GBS is to study the origin and evolution of the population of X-ray binaries. By comparing the
Table 1

Reference Table for the Acronyms used in this Paper

\begin{tabular}{|c|c|}
\hline Acronym & Description \\
\hline ACIS & Advanced CCD Imaging Spectrometer \\
\hline AGN & Active galactic nucleus \\
\hline AM CVn star & AM Canum Venaticorum star \\
\hline$A S C A$ & Advanced Satellite for Cosmology and Astrophysics \\
\hline BB & Black body \\
\hline $\mathrm{BH}$ & Black hole \\
\hline Brems & Bremsstrahlung \\
\hline BSC & Bright source catalog \\
\hline CALDB & Calibration database \\
\hline ChaMPlane & Chandra multi-wavelength plane survey \\
\hline CTIO & Cerro Tololo Inter-American Observatory \\
\hline $\mathrm{CV}$ & Cataclysmic variable \\
\hline EoS & Equation of state \\
\hline GBS & Galactic Bulge Survey \\
\hline \multirow[t]{2}{*}{ GLIMPSE } & Galactic Legacy Infrared Mid-plane \\
\hline & Survey Extraordinaire \\
\hline HMXB & High-mass X-ray binary \\
\hline HR & Hardness ratio \\
\hline HRI & High-resolution imager \\
\hline ID & Identification \\
\hline IP & Intermediate polar \\
\hline LMXB & Low-mass X-ray binary \\
\hline NS & Neutron star \\
\hline PSPC & Position sensitive proportional counter \\
\hline RASS & $R O S A T$ all sky survey \\
\hline RS CVn star & RS Canum Venaticorum star \\
\hline UCXB & Ultra-compact X-ray binary \\
\hline UKIDSS & UKIRT Infrared deep sky survey \\
\hline UKIDSS/GPS & UKIDSS Galactic plane survey \\
\hline VVV & Vista Variables in the Via Lactea \\
\hline W UMa star & W Ursae Majoris star \\
\hline
\end{tabular}

observed number of sources per source class (e.g., cataclysmic variables, CVs and LMXBs) with those that have been predicted on the basis of population synthesis models, one can constrain binary evolution models, in particular the nature of the common envelope phase. Most compact binary sources responsible for high energy phenomena went through one or two phases of common-envelope evolution, such as CVs, AM Canum Venaticorum stars (AM CVns), and UCXBs (Taam \& Sandquist 2000). However that phase is not yet well understood. Compact binaries have much lower orbital energy and angular momentum than the progenitor binary that contained giants (Paczynski 1976). The binary semi-major axis is thought to shrink mainly during a phase of unstable mass transfer and ejection, i.e., the spiral-in. If the outcome of this process is derived by assuming that the change in orbital energy is enough to eject the giant's mantle, the predicted properties do not match the observations of double white dwarf binaries. These properties can be matched with the assumption that the giant's mantle is ejected, carrying a fixed fraction of the total angular momentum (Nelemans et al. 2000), but this begs the question how the required energy is provided. A more complete theoretical description is required that takes into account both energy and angular momentum.

From the properties of single radio pulsars it has been inferred that they receive a kick at birth, due to asymmetries in the supernova explosion (Lyne \& Lorimer 1994). This has important consequences for the evolution and properties of X-ray binaries (e.g., Brandt \& Podsiadlowski 1995; Kalogera 1996) so in principle X-ray binaries can be used to constrain the properties of the kick. In particular, the question is whether BHs also receive a kick at formation and, if so, what its properties are 
(e.g., White \& van Paradijs 1996, Jonker \& Nelemans 2004, Willems et al. 2005, Miller-Jones et al. 2009).

Four main steps must be followed in binary population synthesis modeling (Postnov \& Yungelson 2006). First, a set of initial conditions must be chosen. Key parameters which must be set include the initial mass function, the initial distribution of mass ratios in binaries, the initial binary fraction, and the initial distribution of orbital periods. Second, any evolution with time of these initial conditions, such as that due to changes in star formation rate, should be taken into account. Next, a recipe for (binary) stellar evolution must be specified. The major uncertainties in this recipe include the aforementioned uncertainties in common envelope evolution, uncertainties in the NS and BH natal kick distributions, and uncertainties in mass and angular momentum loss. Finally, a recipe must be developed for converting accretion rates, typically calculated on timescales of centuries or longer, into instantaneous observables such as the X-ray luminosity. Key uncertainties in this step largely involve understanding the spectra of accreting sources at different accretion rates and understanding how disk instabilities will affect the distribution of luminosities of those sources which undergo disk accretion.

To make progress on these issues we envisage a two-pronged approach that includes detailed studies of individual systems on the one hand, and of the population on the other hand. Any viable evolutionary scheme must be able to reproduce the specific properties (such as component masses, orbital period, age, system velocity) of each observed individual system. Any viable evolution scheme must also reproduce the distributions of and correlations between these properties in the population of X-ray binaries. Observationally this requires the discovery of large homogeneous samples of CVs and (ultra-compact) X-ray binaries, and the detailed follow-up of a number of individual systems.

The large number of sources will also allow us to investigate the spatial distribution of LMXBs and this provides input to the LMXB formation scenarios. Jonker \& Nelemans (2004) found that there is a significant excess of LMXBs at $-10^{\circ}<l<0^{\circ}$ with respect to $0^{\circ}<l<10^{\circ}$. Weidenspointner et al. (2008) found an asymmetric distribution of the $511 \mathrm{keV}$ line emission using INTEGRAL data which they suggested was due to an asymmetric LMXB distribution (although see Bandyopadhyay et al. 2009). However, any spatial non-uniformity in the Galactic birth distribution of LMXBs is thought to be washed out by the natal kick imparted on the NS in the LMXB formation models presented by van den Heuvel (1983) and Kalogera (1998). On the other hand, the LMXB evolutionary model, involving triple star evolution, discussed by Eggleton \& Verbunt (1986) provides a channel for the formation of LMXBs without a large kick velocity. In general, except for formation scenarios involving either direct collapse to a $\mathrm{BH}$ or an accretion induced collapse, a Blaauw kick should be imparted regardless of the type of compact object formed during the supernova event (Blaauw 1961). Evidence for velocity kicks is found in the $z$-distribution of LMXBs (van Paradijs \& White 1995; Jonker \& Nelemans 2004) and in the velocity distribution of radio pulsars (Lyne \& Lorimer 1994; Hansen \& Phinney 1997). An improved spatial distribution of LMXBs in the Bulge will help to test if such kicks happen commonly or rarely.

\subsection{Previous X-ray Surveys of the Bulge}

Previous X-ray surveys of the Galactic bulge provide some general expectations for our survey. The Advanced Satellite for Cosmology and Astrophysics (ASCA) observed the central region of our Galaxy with $|l|<45^{\circ}$ and $|b|<0.4$ in the 0.7-10 keV band, albeit with a resolution of $3^{\prime}$ which led to a large number of unclassified sources (Sugizaki et al. 2001). The ASCA survey discovered 163 sources down to a flux of $\approx 3 \times 10^{-12} \mathrm{erg} \mathrm{cm}^{-2} \mathrm{~s}^{-1}$ which as a group have properties in common with CVs, high-mass X-ray binaries (HMXBs), quiescent LMXBs, and Crab-like pulsars. A recent paper by Anderson et al. (2011) finds a number of massive stars within the $A S C A$ Galactic plane sources. The XMM-Newton Galactic plane survey covered $3 \mathrm{deg}^{2}$ along the Galactic plane down to $F_{X}(2-10 \mathrm{keV})$ $2 \times 10^{-14} \mathrm{erg} \mathrm{cm}^{-2} \mathrm{~s}^{-1}$, finding roughly one-third to be soft sources suggestive of nearby coronally active stars (Hands et al. 2004), while two-thirds were hard, absorbed sources. The latter group is dominated by active galactic nuclei (AGNs) but it also has a substantial Galactic component, especially between $10^{-12} \mathrm{erg} \mathrm{cm}^{-2} \mathrm{~s}^{-1}>F_{X}>10^{-13} \mathrm{erg} \mathrm{cm}^{-2} \mathrm{~s}^{-1}$. Optical identifications of 30 sources with $F_{X}>7 \times 10^{-14} \mathrm{erg} \mathrm{cm}^{-2} \mathrm{~s}^{-1}$ led to the classification of $16-18$ coronally active stars, three CVs, and two LMXB candidates (Motch et al. 2010). Finally, spectroscopic follow-up of medium-duration Chandra observations of numerous Galactic plane fields (the ChaMPlane survey, Grindlay et al. 2005; Rogel et al. 2006) finds roughly one-third to be soft sources, largely stars, and two-thirds to be hard sources, including many AGNs and a few CVs.

In this paper, we describe the modeling that provides rough estimates for the number of sources that we expect in the GBS (Section 2). The Chandra X-ray data, the X-ray source list, and analysis of the X-ray source properties are described in Section 3. A brief outline of the multi-wavelength imaging data is given in Section 4, followed by a comparison of ROSAT sources in the GBS area with the Chandra-discovered sources in Section 5. We end with a short summary (Section 6) and outlook (Section 7).

\section{SOURCE NUMBER ESTIMATES}

We used a simple method to roughly estimate the number of X-ray sources in the Bulge area and based on this we devised an optimal observing strategy to best meet our goals. For each source class we used estimates of their typical optical ( $i^{\prime}$-band) and X-ray brightness as well as local space densities or total number of objects in the Galaxy without following their formation or evolution. We distribute these sources in the Galaxy according to the model for the star formation history from Nelemans et al. (2004), which is based on the Milky Way disk formation simulation of Boissier \& Prantzos (1999) with an added Bulge component. We do not take the influence of kicks for NS systems into account. For each object we determine the interstellar absorption from the Schlegel et al. (1998) dust maps, assuming that the dust is evenly distributed (i.e., homogeneous) between the Earth and the position where the line of sight leaves the dust layer for an assumed dust height above and below the Galactic plane of 120 pc. For the optical we use $A_{V}=3.1 E(B-V)$ and $A_{i^{\prime}}=0.6 A_{V}$. We further use $A_{K}=0.1 A_{V}$. For the X-ray absorption we distinguish hard sources (with an assumed power-law spectrum with photon index 2), soft sources (where the assumed spectrum is a black body with temperature $0.25 \mathrm{keV}$ ), and sources with a Bremsstrahlung spectrum with a temperature of $2 \mathrm{keV}$. We link the absorption to the reddening via $N_{\mathrm{H}}=0.179 \times 10^{22} A_{V}$ (Predehl \& Schmitt 1995).

By comparing the expected number of quiescent LMXBs with the number of CVs as a function of X-ray flux (see Figure 2) 


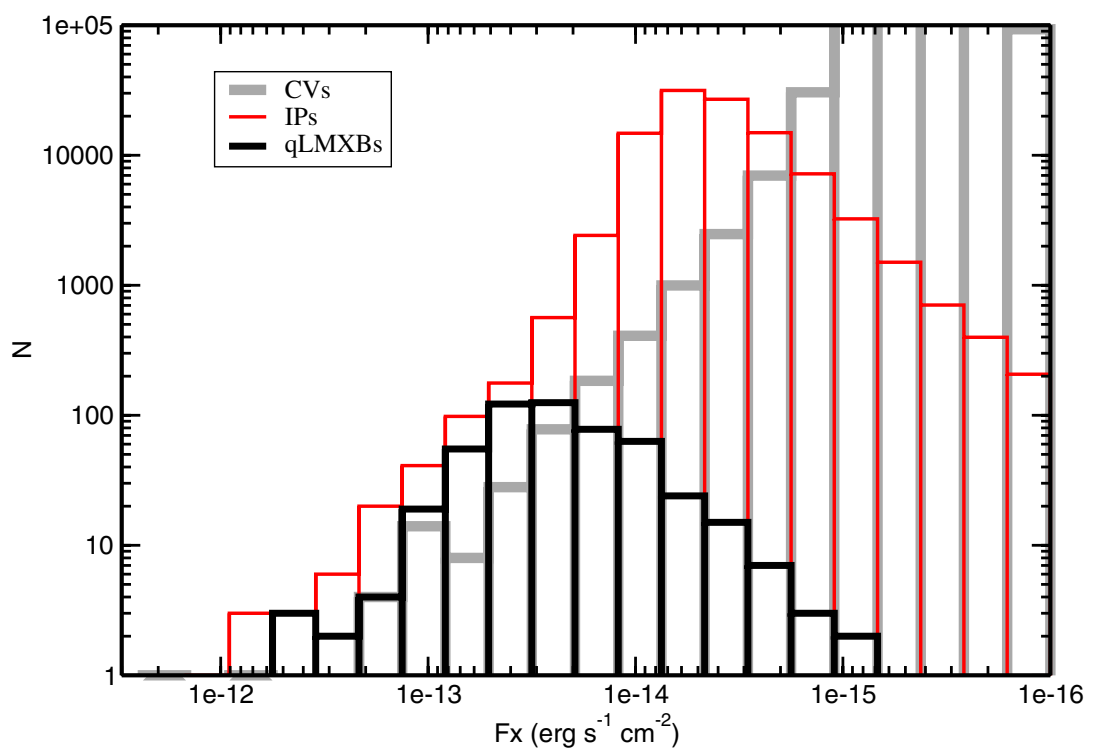

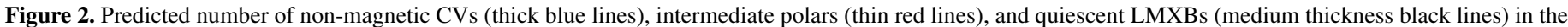

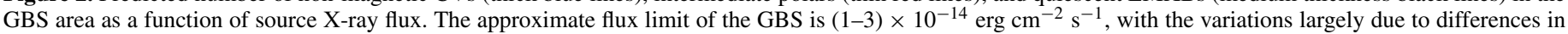

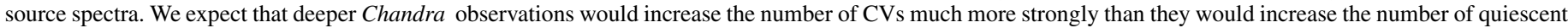
LMXBs.

(A color version of this figure is available in the online journal.)

Table 2

Estimated Numbers of the Various Source Categories

\begin{tabular}{|c|c|c|c|c|c|c|c|c|c|}
\hline (I) & (II) & (III) & (IV) & $(\mathrm{V})$ & (VI) & (VII) & (VIII) & $(\mathrm{IX})$ & $(\mathrm{X})$ \\
\hline LMXB & $10^{35}$ & Hard & 0 & 0 & 140 & 6 & 7 & 7 & 7 \\
\hline qLMXB & $10^{33}$ & $\mathrm{BB}$ & 5 & 2 & 10000 & 120 & 86 & 221 & 532 \\
\hline UCXB & $10^{34}$ & Hard & 4 & 0 & 1000 & 32 & 3 & 56 & 58 \\
\hline qUCXB & $10^{32}$ & Hard & 10 & 0 & 10000 & 1 & 0 & 8 & 605 \\
\hline CV (non mag.) & $10^{31}$ & Brems & 7.5 & 0 & $2 \times 10^{-5}$ & 62 & 61 & 62 & $1.4 \times 10^{6}$ \\
\hline $\mathrm{CV}$ (IP) & $10^{32}$ & Brems & 8.5 & 0 & $1.5 \times 10^{-6}$ & 152 & 5 & 525 & $7.7 \times 10^{4}$ \\
\hline RS CVn & $10^{31}$ & Hard & 2.5 & 1 & $1 \times 10^{-4}$ & 596 & 596 & 596 & $1.3 \times 10^{6}$ \\
\hline W UMa & $5 \times 10^{30}$ & Hard & 4.5 & 2 & $7.5 \times 10^{-5}$ & 160 & 160 & 160 & $2.3 \times 10^{6}$ \\
\hline Be X-ray binaries & $10^{34}$ & Hard & 0 & 0 & 500 & 9 & 9 & 10 & 10 \\
\hline Total & & & & & & 1142 & & 1648 & \\
\hline
\end{tabular}

Notes. The columns describe: the source type (I), the assumed mean X-ray luminosity in erg s ${ }^{-1}$ (II), the X-ray color (III), the assumed mean $i^{\prime}$-band absolute magnitude (IV), the assumed $i^{\prime}-K$ intrinsic color (V), the assumed total number of sources in the Galaxy (integer values) or the space densities $\left(\rho\right.$ pc ${ }^{-3}$ ) (VI), number of sources that should be detectable both in the GBS X-ray as well as the optical survey (VII), the number of sources detected in the GBS X-ray as well as in a $K$-band imaging survey of limiting magnitude of $K=18$ (VIII), the number of sources detected in X-rays in the GBS area (IX), and the number of sources falling in the GBS area (X). Quiescent sources are denoted with "q." "BB" stands for a $0.25 \mathrm{keV}$ "black body." "Brems" stands for Bremsstrahlung.

we decided on rather shallow Chandra observations $(\approx 2 \mathrm{ks}$ per pointing) yielding a limiting flux of (1-3) $\times 10^{-14} \mathrm{erg} \mathrm{cm}^{-2} \mathrm{~s}^{-1}$. This limit is dependent on the source spectrum including extinction. For the source number estimates we take a general flux limit of $3 \times 10^{-14} \mathrm{erg} \mathrm{cm}^{-2} \mathrm{~s}^{-1}$. Deeper observations would mostly increase the number of detected CVs. This would make it more difficult to classify the LMXBs among the sample of $\mathrm{X}$-ray sources. As mentioned above, we selected two strips of $6^{\circ} \times 1^{\circ}$, one centered at 1.5 above and one centered at 1.5 below the Galactic center (see Figure 1). The size and location are chosen in order to detect enough sources in each class to achieve our science goals. The optical extinction is still substantial in this region but relatively low compared to the Galactic center and Galactic plane. This will allow us to obtain optical spectroscopic follow-up to a large fraction of the $\mathrm{X}$-ray sources, which is crucial for achieving our science goals. Going to a higher latitude would facilitate identification as the crowding would be less severe, but the source density drops off quickly, which would require a larger surface area to detect a sufficient number of sources. The chosen strips are thus a compromise between survey area size and location.

The number of sources expected in the GBS area and the assumptions made in the population estimates are given in Table 2 . The values for the assumed X-ray luminosity, $i^{\prime}$-band absolute magnitude, and optical extinction $A_{V}$ in Table 2 are averages. In the modeling we assumed a distribution of X-ray luminosities using a Gaussian distribution, with $\sigma=0.5$ and the average normalized to 1 . For the $i^{\prime}$-band absolute magnitude we apply a Gaussian smoothing with $\sigma=1$ mag. Finally, we use a Gaussian smoothing in the assumed $A_{V}$ with $\sigma=0.2$ mag.

We expect to discover Roche lobe overflow NS and BH $\mathrm{X}$-ray binaries, both with main sequence donors (LMXBs) as well as UCXBs. Most LMXBs will be in quiescence. The numbers for the LMXBs are based on a very simple estimate of $\approx 140$ persistent systems with $L_{\mathrm{X}}>10^{35} \mathrm{erg} \mathrm{s}^{-1}$ in the Galaxy (Grimm et al. 2002), a number ratio of quiescent to persistently 
bright/active LMXBs of $\sim 70$, and the prediction that the number of UCXBs may be equal to the number of non-degenerate LMXBs (Belczynski \& Taam 2004). To estimate the number ratio of quiescent to active LMXBs, we use results of surveys of Galactic globular clusters as input. Roughly 10 times as many quiescent LMXBs are identified (by their soft, blackbody-like spectra) as active LMXBs (Heinke et al. 2003). Since fainter quiescent LMXBs tend to be dominated by their hard, power-law component rather than the blackbody component (e.g., Jonker et al. 2004; Jonker 2008), it has been suggested that half of all quiescent LMXBs are missed in these globular cluster surveys (Heinke et al. 2005). However, it is uncertain whether globular cluster LMXBs are similar in their duty cycles to Galactic LMXBs, as they are formed in different ways.

An alternative way to estimate the number ratio between active and quiescent LMXBs comes from the total number of LMXBs and the estimated X-ray binary lifetime. Portegies Zwart et al. (1997) compute that there are $10^{-5} \mathrm{NS}$ binaries formed per year for most reasonable values of the common envelope parameter. Assuming a typical lifetime as an X-ray binary of approximately $1 \mathrm{Gyr}$, then there are $\approx 10^{4}$ systems in the Galaxy. More recently, Kiel \& Hurley (2006) find a similar number from their evolutionary population synthesis calculations. Taking that there are $\approx 140$ active $X$-ray binaries at any given time, 1 in 70 are active at any given time. We take this as our assumed ratio between active and quiescent LMXBs in our number estimates.

In an X-ray selected sample such as this, another major population of sources that we will pick-up is CVs and of these we will in particular detect magnetic Intermediate Polar systems (IPs). Their number estimate is based on the observed IP and nonmagnetic CV space densities of $\sim 1.5 \times 10^{-6}$ and $2 \times 10^{-5} \mathrm{pc}^{-3}$ (Patterson 1984; Hertz et al. 1990; Pretorius et al. 2007; Rogel et al. 2008). The discovery of a large number of IPs with INTEGRAL (Scaringi et al. 2010) may be indicating that the fraction of IPs among the CVs is substantially larger than was previously thought. In addition, there will be a large number of active (binary) stars such as RS CVn stars. The expected number of background AGNs is 25 (see Ebisawa et al. 2005). We might also find a few ultra-compact double white dwarf systems (AM CVns).

The expected number of W UMa like sources is calculated using the space density of $7.5 \times 10^{-5} \mathrm{pc}^{-3}$ with $M_{i^{\prime}} \approx 4.5$ and an average X-ray luminosity of $5 \times 10^{30} \mathrm{erg} \mathrm{s}^{-1}$ (Rucinski 1998). Finally, the expected number of Be X-ray binaries is estimated using an estimated total number of systems of 500 in our Galaxy, with an $M_{i^{\prime}}=0$ and a hard power-law spectrum with an average luminosity of $L_{X}=1 \times 10^{34} \mathrm{erg} \mathrm{s}^{-1}$. We expect 10 in the GBS area out of which 9 will be detectable in the $i^{\prime}$ band as well as the $K$ band.

These numbers are uncertain and calibrating the number densities of sources using the GBS is one of the principal objectives. We will compare our identifications with the predicted numbers of binaries in each category and thus place strong constraints on important uncertainties in the theory of binary evolution, such as the common-envelope phase and the existence and magnitude of kicks imparted on NSs and BHs.

Of the predicted LMXBs we assume that $10 \%$ will have $\mathrm{BH}$ accretors. This number is highly uncertain but $10 \%$ is roughly the number ratio between $\mathrm{NS}$ and $\mathrm{BH}$ systems observed in HMXBs (see Grimm et al. 2003 and references therein). Some authors have claimed that the ratio between NS and BH systems should be closer to 1 (Romani 1992), whereas others indeed find values closer to $10 \%$ (Portegies Zwart et al. 1997; Kiel \& Hurley 2006). Assuming 10\% would lead to 25 BH LMXBs in the GBS which will approximately double the population of known Galactic BHs for which a dynamical mass measurement is possible (currently 20; McClintock \& Remillard 2006). Eclipsing BH LMXB systems should exist, but have not yet been found. It has been proposed that they are too weak to be detected by current X-ray all sky monitors because they are obscured behind the accretion disk rim during outburst (Narayan $\&$ McClintock 2005). If so, they should turn up in our GBS in quiescence, when the disk is expected to be much thinner. The number of eclipsing sources depends on the distribution of the mass ratio between the accretor and the donor star (see e.g., Horne 1985). For mass ratios $q \approx 0.3$ approximately $20 \%-25 \%$ of the 200 new quiescent LMXBs we expect to discover should be eclipsing of which a handful could be BHs.

Overall, this modeling can be summarized in a figure with $\mathrm{X}$-ray flux as a function of optical $i^{\prime}$-band magnitude for the main components of the expected sources (Figure 3 ). The large majority of bright optical sources with $i^{\prime} \lesssim 16$ associated with an X-ray source in the GBS area is expected to be an RS CVn. Similarly, the majority of X-ray bright sources $\left(F_{X}>1 \times 10^{-12} \mathrm{erg} \mathrm{cm}^{-2} \mathrm{~s}^{-1}\right)$ with an optical counterpart with $18<i^{\prime}<22$ is expected to be an active LMXB or UCXB. Finally, most quiescent LMXBs and UCXBs will have faint optical counterparts $\left(i^{\prime}>21\right)$.

Clearly, other source types are expected, for instance single X-ray active nearby G, K, or M stars (cf. Schmitt \& Liefke 2004) and Algol sources. However, the expected number of sources is small for the Algol and nearby single stars, so we do not provide detailed estimates for these classes.

In order to roughly assess the accuracy of the aforementioned modeling, we compare the number of sources detected in the single, 1 Ms deep Chandra observation of a small part of $20.6 \operatorname{arcmin}^{2}$ of the GBS area presented by Revnivtsev et al. (2009) with the number of sources we predict if one were to observe the whole GBS area to that depth of $1 \times$ $10^{-16} \mathrm{erg} \mathrm{cm}^{-2} \mathrm{~s}^{-1}$. We expect to find 3 million sources based on our source number estimates, whereas scaling the observed $20.6 \operatorname{arcmin}^{2}$ to $12 \mathrm{deg}^{2}$ we would expect to find about 1 million sources. The main difference between these two numbers probably stems from uncertainties in scaling from the $20.6 \mathrm{arcmin}^{2}$ area to a $12 \mathrm{deg}^{2}$ field such as those caused by the difference in extinction between the GBS area with respect to that in the area studied by Revnivtsev et al. (2009), as well as of course uncertainties in our modeling. All in all, the agreement within a factor of three between the number of sources we predict and what was found is not unsatisfactory.

\section{X-RAY OBSERVATIONS}

We have obtained observations with the Chandra X-Ray Observatory (Weisskopf et al. 2002) covering, to date, about two-thirds of the total area of $12 \mathrm{deg}^{2}$ that we envisage for the GBS.

In Figure 1, the wiggles indicate the composite outline of each circular field of view of $14^{\prime}$ diameter of the individual Chandra observations obtained to date covering the GBS area. The Chandra observations have been performed using the I0 to I3 CCDs of the Advanced CCD Imaging Spectrometer (ACIS) detector (Garmire 1997; ACIS-I). The observation identification (ID) numbers for the data presented here are 8643-8774 


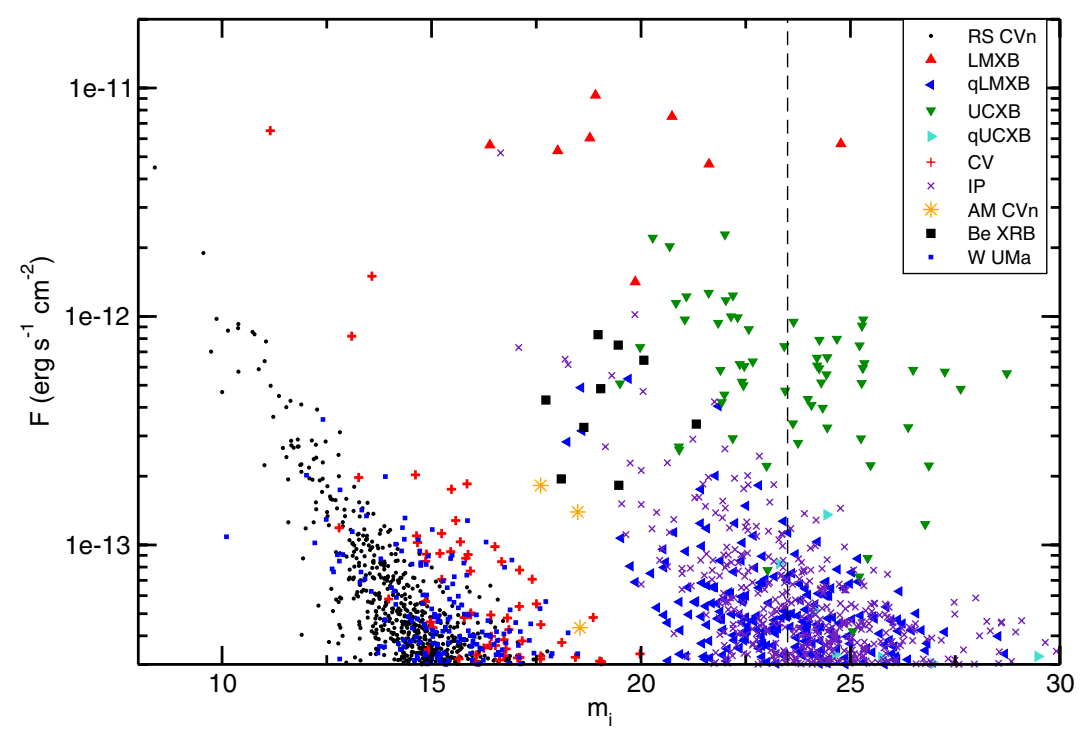

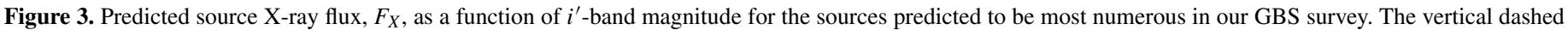
line indicates the approximate $i^{\prime}$-band magnitude limit of our optical imaging.

and 9977-10024. We reprocessed and analyzed the data using the CIAO 4.2 software developed by the Chandra X-ray Center and employing CALDB version 4.3. The data telemetry mode was set to very faint for all observations except that with ID 8687 since there was a bright source present in archival ROSAT observations. For that observation we used the standard faint mode.

We also reprocessed and reanalyzed the data that were obtained as part of the Bulge Latitude Survey (Grindlay et al. 2005; Hong et al. 2009; observation IDs 7160-7162, 7166-7168, 8199-8204, 9562-9564) that falls in the area we target in the GBS using exactly the same reduction and analysis as for our GBS observations. Because the original Bulge latitude survey Chandra observations have an exposure time of approximately $15 \mathrm{ks}$, we selected $2 \mathrm{ks}$ segments to allow for a comparison with our GBS observations. We selected data stretches of $2 \mathrm{ks}$ length taking the start time of the observation from the header of the data, plus $100 \mathrm{~s}$ as the starting point of the $2 \mathrm{ks}$ stretches. These data were also obtained using the very faint mode.

The very faint mode provides $5 \times 5$ pixel information per $\mathrm{X}$-ray event. This allows for a better screening of events caused by cosmic rays. In our analysis we selected events only if their energy falls in the $0.3-8 \mathrm{keV}$ range.

We used WAVDETECT to search for X-ray sources in each of the observations using data covering the full $0.3-8,0.3-2.5$, and $2.5-8 \mathrm{keV}$ energy band separately. We set the SIGTHRESH in WAVDETECT to $1 \times 10^{-7}$, which implies that for a background count rate constant over the ACIS-I CCDs there would be 0.1 spurious source detection per observation as about $1 \times 10^{6}$ pixels are searched per observation. However, as we explain below, we applied additional selection criteria. This lowers the number of spurious sources.

The resulting SIGNI column in the output list of detected sources provided by WAVDETECT is an estimate of the photon flux significance, not of the detection significance. Instead, we retained all sources for which Poisson statistics indicates that the probability of obtaining the number of detected source counts by chance given the expectation for the local background count rate is lower than $1 \times 10^{-6}$. This would be equivalent to a $>5 \sigma$ source detection in Gaussian statistics. Next, we deleted all sources for which WAVDETECT was not able to provide an estimate of the uncertainty on the right ascension $[\alpha]$ or on declination $[\delta]$ as this indicates often that all counts fell in 1 pixel which could well be due to faint afterglow events caused by cosmic ray hits. In addition, we impose a three count minimum for source detection as Murray et al. (2005) simulated that in their XBootes survey with $5 \mathrm{ks}$ ACIS-I exposures, $14 \%$ of the two count sources were spurious (note that this percentage will probably be lower for our GBS exposures of $2 \mathrm{ks}$ ).

Since our Chandra observations were designed to overlap near the edges, we searched for multiple detections of the same source either in one of the energy sub-bands or in the full energy band. We consider sources with positions falling within $3^{\prime \prime}$ of each other likely multiple detections of the same source. Finally, we inspected the source list and found two spurious sources caused by the bright readout trail and piled-up core of source 1 (see Table 3).

In total we detected 1234 distinct sources in the area indicated with circles in Figure 1, including sources detected in the Bulge Latitude Survey area that fall within our GBS area. The source list is given in Table 3 and provides information on $\alpha, \delta$, the error on $\alpha$ and $\delta$, total number of counts detected, the observation ID of the observation resulting in the detection, and the off-axis angle at which the source is detected. The errors on $\alpha$ and $\delta$ are the error provided by WAVDETECT, it does not take into account the typical Chandra bore-sight uncertainty of 0.'6 (90\% confidence).

We provide individual Chandra source names, however, for briefness we use the source number in Table 3 to indicate which source we discuss in this paper. For the error $\sigma_{N}$ on the detected number of counts $N$, Grimm et al. (2005) give $\sigma_{N}=1+\sqrt{N+0.75}$ after Gehrels (1986). To allow for a rough, easy calculation of the source flux based on the detected number of source counts we give the conversion factor for a source spectrum of a power law with a photon index of 2 absorbed by $N_{\mathrm{H}}=1 \times 10^{22} \mathrm{~cm}^{-2}: 7.76 \times 10^{-15} \mathrm{erg} \mathrm{cm}^{-2} \mathrm{~s}^{-1}$ photon $^{-1}$.

\subsection{Sources Detected Multiple Times}

In the tradeoff between a homogeneous survey depth and total survey exposure time we used $7^{\prime}$ as an effective radius of the Chandra field of view in designing the survey. The size and shape of the point-spread function for off-axis angles 
Table 3

The GBS X-ray Source List

\begin{tabular}{|c|c|c|c|c|c|c|c|c|c|c|}
\hline $\begin{array}{l}\text { Source } \\
\text { Name }\end{array}$ & No. & $\begin{array}{c}\alpha \\
(\operatorname{deg})\end{array}$ & $\begin{array}{c}\delta \\
(\mathrm{deg})\end{array}$ & $\begin{array}{l}\text { Error } \alpha \\
(\operatorname{arcsec})\end{array}$ & $\begin{array}{c}\text { Error } \delta \\
(\operatorname{arcsec})\end{array}$ & $\begin{array}{l}\text { No. } \\
\text { (cnt) }\end{array}$ & $\begin{array}{l}\text { Obs } \\
\text { ID }\end{array}$ & $\begin{array}{l}\text { Off-axis } \\
\text { angle }\left({ }^{\prime}\right)\end{array}$ & $\begin{array}{l}\text { No. of } \\
\text { detec. }\end{array}$ & HR \\
\hline CXOGBS J175024.4-290216 & 1 & 267.60182588 & -29.037885415 & 0.034 & 0.011 & 3391 & 8709 & 8.99 & 1 & . \\
\hline CXOGBS J173728.3-290802 & 2 & 264.36831021 & -29.133892744 & 0.058 & 0.084 & 2191 & 8691 & 7.63 & 2 & . \\
\hline CXOGBS J174042.8-281808 & 3 & 265.17839123 & -28.302224391 & 0.048 & 0.033 & 1850 & 8687 & 5.54 & 4 & $\ldots$ \\
\hline CXOGBS J173931.2-290952 & 4 & 264.88008702 & -29.164675805 & 0.104 & 0.072 & 238 & 8679 & 5.63 & 1 & $-0.78 \pm 0.09$ \\
\hline CXOGBS J174009.1-284725 & 5 & 265.03805780 & -28.790455138 & 0.055 & 0.054 & 157 & 8677 & 3.41 & 1 & $0.50 \pm 0.10$ \\
\hline CXOGBS J174445.7-271344 & 6 & 266.19074704 & -27.229022849 & 0.065 & 0.047 & 153 & 8647 & 3.20 & 1 & $-0.25 \pm 0.09$ \\
\hline CXOGBS J173826.1-290149 & 7 & 264.60910401 & -29.030389506 & 0.160 & 0.155 & 150 & 8690 & 6.65 & 2 & $-0.89 \pm 0.12$ \\
\hline CXOGBS J173508.2-292957 & 8 & 263.78448781 & -29.499426909 & 0.119 & 0.073 & 138 & 9997 & 4.78 & 1 & $-0.39 \pm 0.10$ \\
\hline CXOGBS J173508.3-292328 & 9 & 263.78498489 & -29.391224268 & 0.141 & 0.135 & 134 & 9996 & 5.95 & 1 & $-0.64 \pm 0.12$ \\
\hline CXOGBS J173629.0-291028 & 10 & 264.12099622 & -29.174663351 & 0.322 & 0.180 & 122 & 9995 & 8.34 & 3 & $-0.76 \pm 0.16$ \\
\hline
\end{tabular}

Note. The source list provides the GBS source name, the source number as used in this paper, $\alpha$, $\delta$, the error on $\alpha$ and $\delta$, total number of counts detected, the observation ID of the observation resulting in the detection, the off-axis angle at which the source is detected, and the hardness ratio (HR) for sources detected with more than 20 counts. The HR is calculated for the detection where the off-axis angle was smallest if the source was detected multiple times.

(This table is available in its entirety in a machine-readable form in the online journal. A portion is shown here for guidance regarding its form and content.)

larger than $7^{\prime}$ have degraded such that many optical and/or near-infrared stars will fall inside the X-ray error circle, even for sources detected with more than 10 counts, making multiwavelength follow-up more difficult (cf. Hong et al. 2005). As noted above, this yields the possibility of multiple detection of sources discovered in the overlap regions. Indeed, 105 sources are detected more than once, where we have taken source positions within $3^{\prime \prime}$ of each other as multiple detections of the same source. Out of these 105 sources, 95 sources are detected two times, 9 sources are detected three times, and 1 source is detected four times. The properties that we list in Table 3 for these sources are those of the detection that gave rise to the largest number of X-ray counts. In Table 3 we also list the number of times that sources are detected. In addition, we found that although Chandra sources 33 and 230 are 4". 8 apart, they are conceivably two detections of the same source. Both detections are rather far off-axis (5'.2 and 8.8, respectively) explaining the relatively large uncertainty in both measurements of the position.

\subsection{Detection Probability versus Off-axis Angle}

We investigate the relationship between the number of sources detected as a function of off-axis angle and the number of source counts. In this way we can determine the approximate number of source counts where the survey sensitivity for off-axis angles less than $7^{\prime}$ is constant. We find (Figure 4) that when normalized to the surface area, the number of detected sources drops as a function of off-axis angle for angles larger than $\approx 2^{\prime}$. The probable reason for the slightly lower number of sources detected per unit surface area inside the $2^{\prime}$ radius is that the chip gaps between the four ACIS-I CCDs subtend a larger fraction of the total solid angle within $2^{\prime}$ than they do in the outer regions. That area has a lower effective exposure time as can be seen in Figure 5. For source counts larger than 10 the normalized number of detected sources is approximate constant for off-axis angles less than 7' (blue dot-dashed line in Figure 4). Thus, the GBS coverage is approximately homogeneous down to source fluxes of $\approx 7.7 \times 10^{-14} \mathrm{erg} \mathrm{cm}^{-2} \mathrm{~s}^{-1}$, whereas the sensitivity is decreasing with off-axis angle for fainter sources.

\subsection{X-ray Spectral Information}

We extract source counts using circular source extraction regions of $10^{\prime \prime}$. Background extraction regions are annuli with

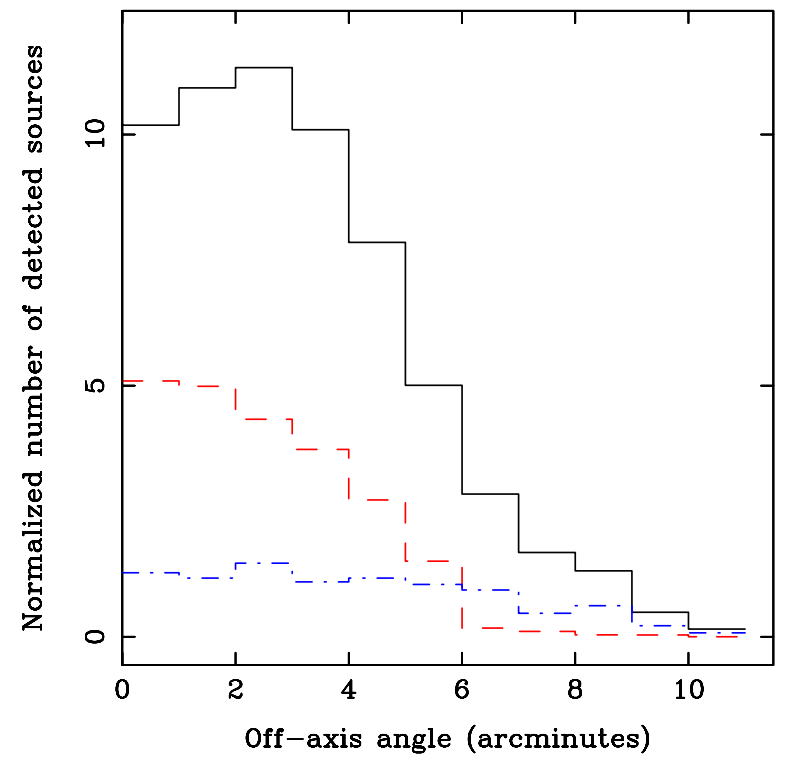

Figure 4. Number of detected sources normalized to area on the sky as a function of off-axis angle. The black solid line indicates all sources, the red dashed line indicates sources detected with only three X-ray counts, and the blue dot-dashed lines are sources detected with 10 counts or more. From the black and red lines it is clear that the survey sensitivity to low count sources is higher when nearer to on-axis, as expected. The fact that the blue line is approximately flat up to $7^{\prime}$ indicates that the GBS survey sensitivity is approximate constant for sources of 10 counts or more. For a power-law spectrum with photon index of 2 and an $N_{\mathrm{H}}=1 \times 10^{22} \mathrm{~cm}^{-2}$ this corresponds to a flux of $7.7 \times 10^{-14} \mathrm{erg} \mathrm{cm}^{-2} \mathrm{~s}^{-1}$.

(A color version of this figure is available in the online journal.)

inner and outer radii of $15^{\prime \prime}$ and $30^{\prime \prime}$, respectively. We plot the 89 sources for which we detected more than 20 counts in a hardness-intensity diagram (Figure 6). To mitigate the effects that small differences in exposure time across our survey can have, we use count rates as a measure of intensity. We define the hardness ratio as the ratio between the count rate in the $2.5-8 \mathrm{keV}$ minus that in the $0.3-2.5 \mathrm{keV}$ band to the count rate in the full $0.3-8 \mathrm{keV}$ energy band (after Kim et al. 2004). We derived the hardness using XSPEC version 12.6 (Arnaud 1996) by determining the count rates in the soft and hard bands taking the response and ancillary response file for each of the sources. The three brightest sources suffer from photon pileup, where more than one photon is registered during the CCD 


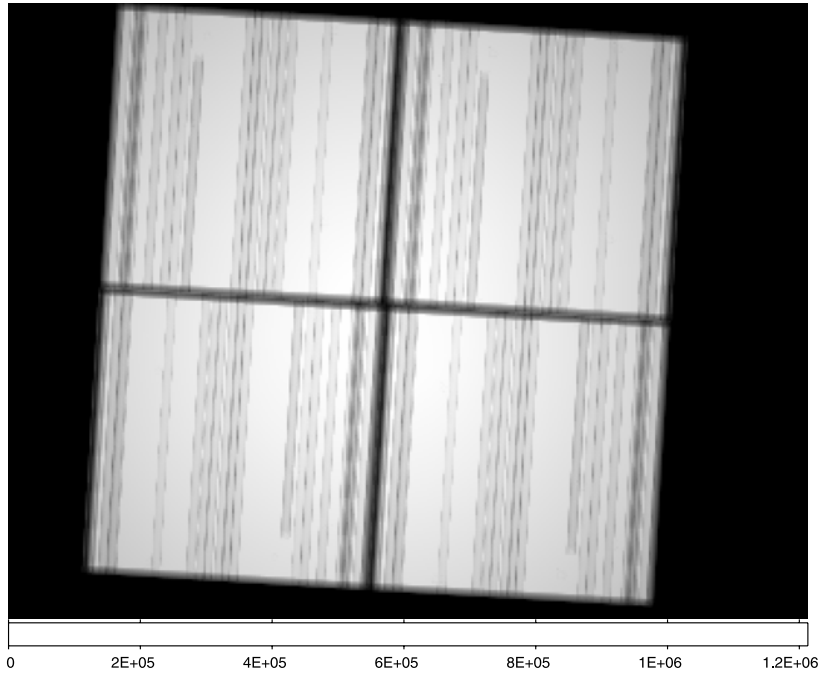

Figure 5. Exposure map for the observation with Obs ID 9999 for an assumed mono-chromatic source emitting $1 \mathrm{keV}$ photons, showing the lower exposure for the gaps between the ACIS I CCDs. Lighter areas have a higher exposure on the sky (in terms of $\mathrm{cm}^{2} \mathrm{~s}^{-1}$ ). Due to the satellite dithering some exposure is achieved even for the gaps. The vertical strips are caused by the presence of columns of pixels of reduced efficiency. The gray area is approximately $8^{\prime} \times 8^{\prime}$ in size.

integration time (see, e.g., Davis 2001). Photon pileup will artificially harden those three sources in a hardness-intensity diagram, therefore, we have not plotted these three sources in Figure 6 nor in Table 3. For the other 86 detected sources photon pileup is unimportant (less than a few percent). Naively, one would expect most hard sources to be more distant and more reddened than the soft sources, as the intrinsic spectral shape of the most numerous classes of sources we expect to find does not differ much.

The most interesting aspect from Figure 6 is perhaps the paucity of bright hard sources. Conversely, most bright sources are probably nearby with low extinction. As foreseen, the spectral information is insufficient for source classification for the majority of the total number of detected sources, therefore, classification will have to come from multi-wavelength observations. Finally, there seems to be a dichotomy in the hardness with one peak centered on a hardness of 0.4 and another centered on -0.5 with a clear paucity of sources with hardness 0 . A similar dichotomy was reported in Warwick et al. (2010).

A conceivable explanation for the nature of this dichotomy is that the soft sources are dominated by nearby sources, such as RS CVns. The harder sources are further away and are either intrinsically hard spectrum sources or they appear hard due to the effects of extinction. The latter increases strongly toward the Galactic center for distances between $\approx 3$ and $5 \mathrm{kpc}$ (Marshall et al. 2006). The strong increase in extinction over a small distance interval would, in this scenario, be responsible for the lack of sources near a hardness of 0 .

\section{MULTI-WAVELENGTH OBSERVATIONS}

Optical imaging observations covering the full GBS area have been obtained using the prime focus MOSAIC II instrument mounted on the $4 \mathrm{~m}$ Victor M. Blanco telescope at the Cerro Tololo Inter-American (CTIO) observatory. We employed the Sloan $r^{\prime}, i^{\prime}$, and $\mathrm{H} \alpha$ filters with exposures of 120,180 , and $480 \mathrm{~s}$, respectively.

For the optical astrometry we used the UCAC2 catalog (Zacharias et al. 2004). The UCAC2 is made up by stars brighter

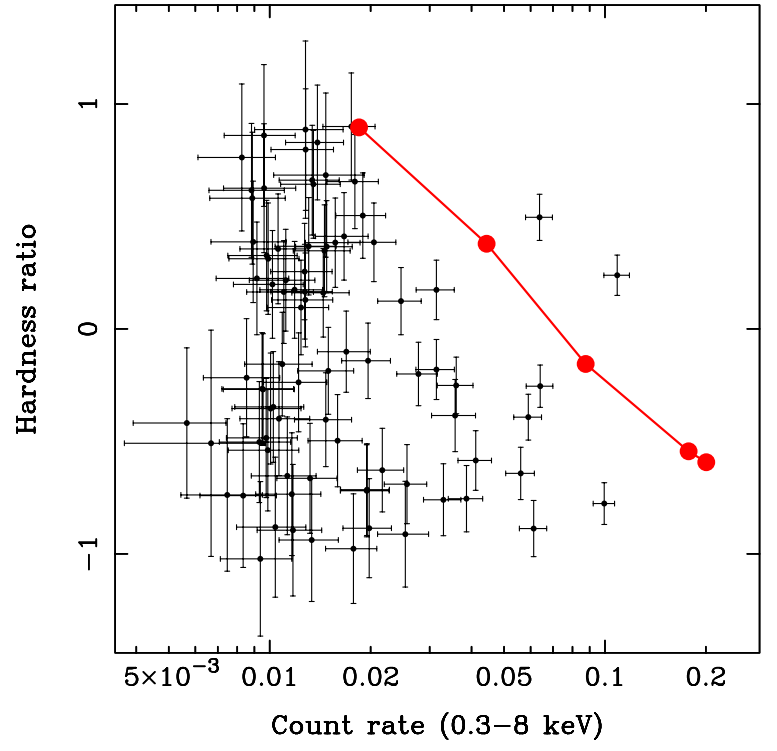

Figure 6. Hardness-intensity diagram for the 86 sources for which more than 20 counts were detected in the GBS survey (we exclude the three brightest sources from the plot since their hardness ratio is strongly affected by effects of photon pileup). To mitigate effects of small differences in exposure times we used count rates as a measure of intensity. The hardness is defined as the ratio between the count rate in the $2.5-8 \mathrm{keV}$ minus that in the $0.3-2.5 \mathrm{keV}$ band to the count rate in the full $0.3-8 \mathrm{keV}$ energy band. Hard sources fall in the top half and soft sources in the bottom half of this figure. The red line with large bullets shows the influence of the extinction $N_{\mathrm{H}}$ on a power-law spectrum with index 2 for a source count rate of 0.2 counts s$~^{-1}$ and $N_{\mathrm{H}}$ of $0.01 \times 10^{22} \mathrm{~cm}^{-2}$. The $N_{\mathrm{H}}$ is increasing from bottom right to top left from $(0.01,0.1,1,3,10) \times 10^{22} \mathrm{~cm}^{-2}$. (A color version of this figure is available in the online journal.)

than 16th magnitude that would saturate in the $120 \mathrm{~s}$ long $r^{\prime}$ band observations. Therefore, to facilitate accurate astrometric calibration of the optical observations we have also obtained short, $10 \mathrm{~s}$ long, $r^{\prime}$-band exposures for each pointing. A detailed discussion of the optical observations and the findings will be presented in a forthcoming paper (C. G. Bassa et al. 2011, in preparation).

Using the same telescope and instrument (Blanco and MOSAIC II), we re-imaged the fields of the initial optical observations at random intervals over eight nights in the Sloan $r^{\prime}-$ band. The goal of these observations is to search for variability induced by binary motion. A detailed discussion of these optical variability observations and the findings will be presented in a forthcoming paper (R. I. Hynes et al. 2011, in preparation).

In addition to the optical observations, the survey field has also partially been observed in the near-infrared as part of the UKIRT Infrared Deep Sky Survey (UKIDSS) Galactic Plane Survey (UKIDSS/GPS; Lucas et al. 2008). Furthermore, the GBS area is fully covered as part of the Vista Variables in the Via Lactea (VVV) survey (Minniti et al. 2010; S. Greiss et al. 2011, in preparation). These near-infrared observations will also help us distinguishing between the different expected source types. Finally, the Spitzer Galactic Legacy Infrared Mid-plane Survey Extraordinaire (GLIMPSE; Churchwell et al. 2009) survey has covered the GBS area, providing mid-infrared imaging of the survey area.

We have also started with the optical spectroscopic identification of the detected X-ray sources using a suite of optical telescopes such as the Blanco telescope, the New Technology Telescope, the Magellan telescopes, the Gemini-South telescope, and the Very Large Telescope. Results of these observations will also be presented in forthcoming papers. 


\section{COMPARISON WITH ROSAT ALL SKY SURVEY SOURCES}

In order to investigate whether bright sources in our source list are detected by the ROSAT All Sky Survey (RASS; Voges et al. 1999) and, conversely, to investigate if bright RASS sources are still detected in our Chandra observations, we searched for ROSAT sources within $30^{\prime \prime}$ of our Chandra positions.

\subsection{Persistent Sources among Chandra Sources 1-10}

The brightest Chandra source, source 1 is a transient (see below). The four ROSAT Bright Source Catalog (BSC) sources, 1RXS J173728.0-290759, 1RXS J174043.1-281806, 1RXS J173933.4-291001, 1RXS J173826.7-290140, probably correspond to Chandra source number 2, 3, 4, and 7 in Table 3, as the nominal offset between the Chandra and ROSAT position is $5^{\prime \prime} .76,44^{\prime \prime} 26,29$.'7, and $11^{\prime \prime} .6$, respectively. The ROSAT positional $(1 \sigma)$ uncertainty of these four sources is $9^{\prime \prime}, 8^{\prime \prime}, 13^{\prime \prime}$, and 16", respectively. Source 2 of the Chandra GBS survey (1RXS J173728.0-290759) is a well-known Seyfert 1 galaxy. Source 3 (1RXS J174043.1-281806) is the persistent LMXB and UCXB-candidate SLX 1737-282. Source 4 is associated with HD 316072; the angular distance between the Chandra position and HD 316072 is 0.46 . HD 316072 is a bright, $V=9.92$, G9III star. This source is conceivably a bright active star or a long-period LMXB. Taking the observed and absolute $V$-band magnitude and assuming $A_{V}=0$ yields a maximum source distance of $675 \mathrm{pc}$. Modeling the Chandra X-ray spectrum consisting of 238 photons, we find that the source spectrum is soft. It can be described by an absorbed black body spectrum with $k T=$ $0.22 \mathrm{keV}$ and $N_{\mathrm{H}}=0.4 \times 10^{22} \mathrm{~cm}^{-2}$. This provides a good fit at an absorbed/unabsorbed $0.5-10 \mathrm{keV}$ flux of $5.6 \times 10^{-13} /$ $1.2 \times 10^{-12} \mathrm{erg} \mathrm{cm}^{-2} \mathrm{~s}^{-1}$, respectively. This converts to a $0.5-10 \mathrm{keV}$ luminosity upper limit of $6.5 \times 10^{31} \mathrm{erg} \mathrm{s}^{-1}$. Such a soft spectrum would be consistent both with a quiescent NS LMXB as well as a white dwarf accretor in a CV. Optical time series spectroscopy should allow us to distinguish between an active (binary) star or an X-ray binary scenario. Source 7 is 0 '.34 away from a bright optical source identified as a pre-mainsequence star by Torres et al. (2006).

Similarly, sources 5, 6, 8-10 are bright enough that they should have been detected by the RASS BSC. Source 5 is 2 ".78 away from AX J1740.1-2847, which is identified as a lowluminosity high-mass X-ray binary pulsar (Kaur et al. 2010). Source 6 is a known Be X-ray binary which is listed in the RASS faint source catalog as 1RXS J174444.7-271326, indicating some moderate X-ray variability. Source 8 is nominally 29 '.8 away from AX J1735.1-2930, which is currently unclassified. Source 9 is 0.'33 away from HD315997, which is an A5 star in a known eclipsing binary in a $2.8723 \mathrm{~d}$ orbital period (Nesterov et al. 1995; Otero et al. 2006). The $V$-band magnitude varies between 11.21 and 11.40 with a secondary eclipse minimum of 11.24 (Otero et al. 2006). The small size of a NS and BH implies that an accretion disk should be present if one wants to explain the amplitude and duration of the secondary eclipse in an $\mathrm{X}$-ray binary context. Taking the observed and absolute $V$-band magnitude and assuming $A_{V}=0$ yields a maximum source distance of $695 \mathrm{pc}$ for the A5 star. This implies a maximum source $0.5-10 \mathrm{keV}$ luminosity of $\approx 6 \times 10^{31} \mathrm{erg} \mathrm{s}^{-1}$. It is also possible that the A5 star is in orbit with a late type star that is not seen in the optical spectrum but which is responsible for the X-ray emission. Again, orbital phase resolved spectroscopic observations are necessary to reveal the nature of this object.

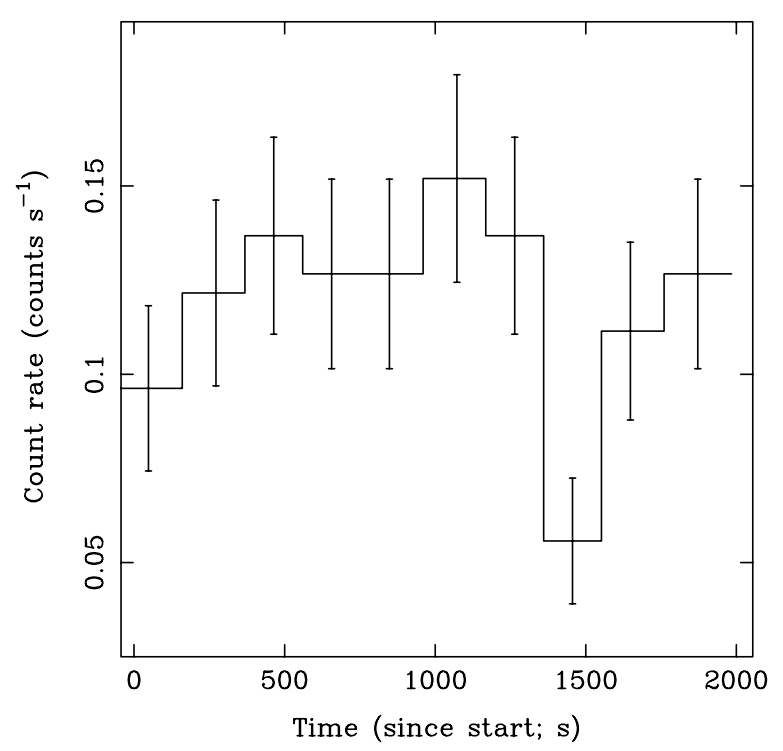

Figure 7. Light curve with a $200 \mathrm{~s}$ bin size of source 4 from Table 3 . There is marginal evidence for a dip/eclipse like feature near $T=1500 \mathrm{~s}$, although the $\chi^{2}$ value of a fit of a constant is 27.5 for 20 degrees of freedom.

Source 10 is 0.47 away from HD $315992(\mathrm{~F} 8, V=9.98)$ and nominally 26".38 away from 1RXS J173628.8-291055 which is listed in the RASS faint source catalog. In the ASCA Galactic center survey the source is found to have a $0.7-10 \mathrm{keV}$ flux of $1 \times 10^{-12} \mathrm{erg} \mathrm{cm}^{-2} \mathrm{~s}^{-1}$ (Sakano et al. 2002). As for several of the other sources discussed above, multiple optical spectra of this source will allow us to investigate if they are in a binary and, if so, what the nature of the second star is.

To summarize, the Chandra point sources 2-10 correspond to: the Seyfert 1 galaxy 1RXS J173728.0-290759, SLX 1737-282, HD 316072, AX J1740.1-2847, 1RXS J174444.7-271326, 1RXS J173826.7-290140, AX J1735.12930, HD 315997, and HD 315992.

\subsubsection{Chandra Light Curves}

We inspect the Chandra light curves of sources 1-10. Sources 1,3 , and 5 show evidence for flare-like variability. Fitting the light curve with a constant gives a $\chi^{2}$ value of 359,57 , and 43 for 20,17 , and 20 degrees of freedom, respectively.

There is marginal evidence in the light curve of source 4 for the presence of an eclipse-like feature (see Figure 7), although, fitting the light curve with a constant gives a $\chi^{2}$ value of 27.5 for 20 degrees of freedom.

The light curves of sources $2,6,7,8,9$, and 10 are consistent with being constant with $\chi^{2}$ values of $24.3,27.2,22.7,27.4$, 12.4 , and 20.5 for 20 degrees of freedom, respectively (except source 9 for which there are 19 degrees of freedom).

\subsection{Transient Sources}

The only other two BSC RASS sources in the GBS area, 1RXS J175113.2-293842 and 1RXS J174220.8-273736, are not detected in our GBS survey. Their nominal ROSAT positional errors are $26^{\prime \prime}$ and $12^{\prime \prime}$, respectively. This means that, especially for 1RXS J175113.2-293842, it is not inconceivable that the source has a Chandra counterpart further away than $30^{\prime \prime}$. However, even in a search radius of $1^{\prime}$ there is no Chandra source

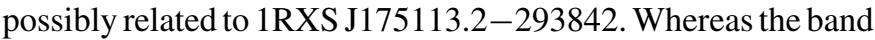
passes of ROSAT (0.1-2.5 keV) and Chandra (0.3-8 keV) are different, Chandra is much more sensitive and both sources 
Table 4

Sources from Pointed ROSAT Observations that Fall in the GBS Area

\begin{tabular}{|c|c|c|c|}
\hline ROSAT name & Chandra & $\begin{array}{c}\alpha \\
\text { (hh:mm:ss) }\end{array}$ & $\begin{array}{c}\delta \\
\text { (dd:mm:ss) }\end{array}$ \\
\hline 1RXH J173547.0-302851 ${ }^{\mathrm{a}}$ & \# 43 & $17: 35: 45.53$ & $-30: 29: 00.0$ \\
\hline 1RXH J173803.6-290706 & \# 31 & $17: 38: 03.50$ & $-29: 07: 06.1$ \\
\hline 2RXP J173940.7-285116 & \# 115 & $17: 39: 40.80$ & $-28: 51: 11.7$ \\
\hline 2RXP J174046.7-283849 & \# 469 & $17: 40: 46.58$ & $-28: 38: 50.6$ \\
\hline 2RXP J174104.6-281504 ${ }^{\mathrm{a}}$ & \# 32 & 17:41:04.91 & $-28: 15: 03.4$ \\
\hline 2RXP J174133.7-284035 & \# 21 & $17: 41: 33.76$ & $-28: 40: 33.8$ \\
\hline 2RXP J174141.9-283324a & \# 114 & $17: 41: 41.76$ & $-28: 33: 24.2$ \\
\hline 2RXP J174249.9-275028 & \# 785 & $17: 42: 49.95$ & $-27: 50: 38.5$ \\
\hline 2RXP J174834.7-295730 & $\# 230^{\mathrm{b}}$ & $17: 48: 35.26$ & $-29: 57: 31.9$ \\
\hline 2RXP J174834.7-295730 & $\# 33^{\mathrm{b}}$ & $17: 48: 35.54$ & $-29: 57: 28.8$ \\
\hline 2RXP J174928.4-291901 ${ }^{\mathrm{a}}$ & \# 156 & $17: 49: 28.31$ & $-29: 18: 59.4$ \\
\hline 2RXP J175029.3-285954 & \# 13 & $17: 50: 29.13$ & $-29: 00: 02.3$ \\
\hline 2RXP J175041.2-291644a & \# 183 & $17: 50: 41.17$ & $-29: 16: 44.5$ \\
\hline
\end{tabular}

Notes.

a Multiple sources from the ROSAT pointed source catalog are consistent with the Chandra position. We list the nearest one.

b These two Chandra sources are 4.' 8 apart; these are likely two detections of the same Chandra source.

should have been exceptionally soft for it not to be detected in Chandra; this is not the case. The ROSAT hardness ratio 1 (HR1) for 1RXS J175113.2-293842 is $1.0 \pm 0.3$ and hardness ratio 2 $(\mathrm{HR} 2)$ is $0.54 \pm 0.27$ (where $\mathrm{HR} 1=(\mathrm{B}-\mathrm{A}) /(\mathrm{B}+\mathrm{A})$ and $\mathrm{HR} 2=$ $(\mathrm{D}-\mathrm{C}) /(\mathrm{D}+\mathrm{C})$, with $\mathrm{A}=0.11-0.41 \mathrm{keV}, \mathrm{B}=0.52-2.0 \mathrm{keV}$, $\mathrm{C}=0.5-0.9 \mathrm{keV}$, and $\mathrm{D}=0.9-2.0 \mathrm{keV}$ count rate). Assuming a power-law spectrum with index 2 , an interstellar extinction of $1 \times 10^{22} \mathrm{~cm}^{-2}$ (consistent with the observed hardness ratios HR1 and HR2) and using the RASS-measured $0.1-2.4 \mathrm{keV}$ count rate of 0.07 counts $\mathrm{s}^{-1}$, we calculate the absorbed $0.1-2.4 \mathrm{keV}$ flux to be approximately $1 \times 10^{-12} \mathrm{erg} \mathrm{cm}^{-2} \mathrm{~s}^{-1}$ at the time of the ROSAT observation. Assuming Chandra detected zero counts and following Gehrels (1986), we take an upper limit of 3 counts for a $95 \%$ upper limit over the $2 \mathrm{ks}$ exposure. This makes the approximate Chandra $0.1-2.4 \mathrm{keV}$ upper limit for such a source spectrum $5 \times 10^{-15} \mathrm{erg} \mathrm{cm}^{-2} \mathrm{~s}^{-1}$, implying a decay in flux of at least a factor 200 .

For 1RXS J174220.8-273736 the detected RASS count rate is 0.16 counts $\mathrm{s}^{-1}$, the HR1 is $1.00 \pm 0.03$ and the HR2 is $1.00 \pm 0.08$. For the same assumed source spectrum as above the absorbed 0.1-2.4 keV ROSAT flux was $3 \times 10^{-12} \mathrm{erg} \mathrm{cm}^{-2} \mathrm{~s}^{-1}$, implying that the source decayed in flux more than a factor of 500 .

Conversely, the brightest source in our survey, SAX J1750.8-2900 (Natalucci et al. 1999), was not detected in the RASS BSC. This source is a recurrent transient LMXB that happened to be in outburst when our Chandra observation was obtained but that was apparently in quiescence during the RASS observations.

\subsection{Sources from Pointed ROSAT Observations}

We list ROSAT sources discovered in pointed observations when they fall inside the covered GBS area. Sources found using both the position sensitive proportional counter (PSPC) detector as well as those found using the high-resolution imager (HRI) observations are included (see Table 4). We exclude pointed observations of sources discussed above (Chandra source numbers 1-10).
Sometimes multiple ROSAT observations of the same source region provide slightly different source coordinates, and thus different ROSAT names, whereas using Chandra we find one source. Although it is possible that there was indeed more than one source and these have faded below the Chandra detection level, it is more likely that these multiple ROSAT detections are in fact of one and the same source. As an example, the six ROSAT sources 2RXP J173826.2-290147, 2RXP J173827.4-290138, 2RXP J173827.5-290152, 2RXP J173826.7-290205, 2RXP J173827.2-290144, 2RXP J173824.0-290146 are probably all related to Chandra source 7 (at $\alpha=17: 38: 26.18$ and $\delta=-29: 01: 49.4)$. The nominal angular distance between the ROSAT positions and the single Chandra position is 2".45, $18^{\prime \prime} .4,16^{\prime \prime} 8,16^{\prime \prime} 7,14^{\prime \prime}$, and 28.'3, respectively. The last ROSAT position was derived from an observation that had the source far off-axis (50'8). Similarly, the smallest positional offset was obtained for the observation where the source was only $14^{\prime} .8$ off-axis.

\section{SUMMARY}

We have started the Chandra GBS with the goals: (1) to identify quiescent, eclipsing NS and BH X-ray binaries that are bright enough in the optical or near-infrared to allow phase resolved spectroscopic observations tailored to measure the compact object mass and (2) to constrain binary population synthesis and X-ray binary formation and evolution models by means of a source number count and a study of the spatial distribution of X-ray binaries.

In this paper, we have presented the Chandra source list and some properties of the X-ray sources of observations covering $\sim$ two-thirds $\left(\approx 8.3 \mathrm{deg}^{2}\right)$ of the total envisaged survey area of $12 \mathrm{deg}^{2}$. The accurate Chandra source position will help identify the optical counterparts. The 1234 X-ray sources that have been discovered so far compare well with the total number of $\approx 1650 \mathrm{X}$-ray sources that we predict should detect in the full $12 \mathrm{deg}^{2}$. However, this is of course no guarantee that the number of sources per source class are close to those we calculated.

We compared our source list with the source list of the RASS. Two BSC RASS sources, 1RXS J175113.2-293842 and 1RXS J174220.8-273736, are not detected in our GBS survey, indicating a decrease in flux with factors larger than 200 and 500, respectively. Furthermore, we compared our Chandra source list with the sources found in the catalog of sources derived from pointed HRI and PSPC ROSAT observations that fall inside the GBS area (see Table 3).

We also imaged the complete survey area using optical observations obtained with the $4 \mathrm{~m}$ Blanco telescope at CTIO. Furthermore, we re-imaged the survey area $\approx 30$ times in the $r^{\prime}$ band to search for (periodic) variable sources. Results of these campaigns will be presented in forthcoming papers.

\section{OUTLOOK}

We are in the process of obtaining optical spectroscopic and photometric observations of all optical proposed counterparts in the Chandra error circle to classify the X-ray sources. This step is crucial to achieve our science goals outlined above.

A full discussion of all known information on all the $\mathrm{X}$-ray sources is beyond the scope of this paper and we defer the discussion of the other fainter Chandra sources to a forthcoming paper, where they will be discussed together with optical photometric and spectroscopic information. 
P.G.J. and G.N. acknowledge support from the Netherlands Organisation for Scientific Research. R.I.H., C.T.B, V.J.M., and L.G. acknowledge support from National Science Foundation Grant No. AST-0908789 and NASA/Louisiana Board of Regents grant NNX07AT62A/LEQSF(2007-10) Phase3-02. C.O.H. is supported by NSERC. D.S. acknowledges an STFC Advanced Fellowship. T.J.M. and A.D. thank STFC for support via a rolling grant to the University of Southampton.

\section{REFERENCES}

Anderson, G. E., et al. 2011, ApJ, 727, 105

Arnaud, K. A. 1996, in ASP Conf. Ser. 101, Astronomical Data Analysis Software and Systems V, Vol. 5, ed. G. H. Jacoby \& J. Barnes (San Francisco, CA: ASP), 17

Bandyopadhyay, R. M., Silk, J., Taylor, J. E., \& Maccarone, T. J. 2009, MNRAS, 392,1115

Belczynski, K., \& Taam, R. E. 2004, ApJ, 603, 690

Blaauw, A. 1961, Bull. Astron. Inst. Neth., 15, 265

Boissier, S., \& Prantzos, N. 1999, MNRAS, 307, 857

Brandt, N., \& Podsiadlowski, P. 1995, MNRAS, 274, 461

Cantrell, A. G., et al. 2010, ApJ, 710, 1127

Churchwell, E., et al. 2009, PASP, 121, 213

Davis, J. E. 2001, ApJ, 562, 575

Ebisawa, K., et al. 2005, ApJ, 635, 214

Eggleton, P. P., \& Verbunt, F. 1986, MNRAS, 220, 13P

Garmire, G. P. 1997, BAAS, 29, 823

Gehrels, N. 1986, ApJ, 303, 336

Grimm, H., Gilfanov, M., \& Sunyaev, R. 2002, A\&A, 391, 923

Grimm, H., Gilfanov, M., \& Sunyaev, R. 2003, MNRAS, 339, 793

Grimm, H., McDowell, J., Zezas, A., Kim, D., \& Fabbiano, G. 2005, ApJS, 161 , 271

Grindlay, J. E., et al. 2005, ApJ, 635, 920

Hands, A. D. P., Warwick, R. S., Watson, M. G., \& Helfand, D. J. 2004, MNRAS, 351,31

Hansen, B. M. S., \& Phinney, E. S. 1997, MNRAS, 291, 569

Heinke, C. O., Grindlay, J. E., \& Edmonds, P. D. 2005, ApJ, 622, 556

Heinke, C. O., Grindlay, J. E., Lugger, P. M., Cohn, H. N., Edmonds, P. D., Lloyd, D. A., \& Cool, A. M. 2003, ApJ, 598, 501

Hertz, P., Bailyn, C. D., Grindlay, J. E., Garcia, M. R., Cohn, H., \& Lugger, P. M. 1990, ApJ, 364, 251

Hong, J. S., van den Berg, M., Grindlay, J. E., \& Laycock, S. 2009, ApJ, 706, 223

Hong, J. S., van den Berg, M., Schlegel, E. M., Grindlay, J. E., Koenig, X., Laycock, S., \& Zhao, P. 2005, ApJ, 635, 907

Horne, K. 1985, MNRAS, 213, 129

Jonker, P. G. 2008, in AIP Conf. Ser. 983, 40 Years of Pulsars: Millisecond Pulsars, Magnetars and More, ed. C. Bassa, Z. Wang, A. Cumming, \& V. M. Kaspi (New York: AIP), 519

Jonker, P. G., \& Nelemans, G. 2004, MNRAS, 354, 355

Jonker, P. G., Wijnands, R., \& van der Klis, M. 2004, MNRAS, 349, 94

Kalogera, V. 1996, ApJ, 471, 352

Kalogera, V. 1998, ApJ, 493, 368

Kaur, R., Wijnands, R., Paul, B., Patruno, A., \& Degenaar, N. 2010, MNRAS, 402,2388

Kiel, P. D., \& Hurley, J. R. 2006, MNRAS, 369, 1152

Kim, D., et al. 2004, ApJS, 150, 19

Lattimer, J. M., \& Prakash, M. 2004, Science, 304, 536

Lucas, P. W., et al. 2008, MNRAS, 391, 136

Lyne, A. G., \& Lorimer, D. R. 1994, Nature, 369, 127

Marshall, D. J., Robin, A. C., Reylé, C., Schultheis, M., \& Picaud, S. 2006, A\&A, 453,635
Mauerhan, J. C., Muno, M. P., Morris, M. R., Bauer, F. E., Nishiyama, S., \& Nagata, T. 2009, ApJ, 703, 30

McClintock, J. E., \& Remillard, R. A. 2006, in Black Hole Binaries, ed. W. Lewin \& M. van der Klis (Cambridge: Cambridge Univ. Press), 157

Miller-Jones, J. C. A., Jonker, P. G., Nelemans, G., Portegies Zwart, S., Dhawan, V., Brisken, W., Gallo, E., \& Rupen, M. P. 2009, MNRAS, 394, 1440

Minniti, D., et al. 2010, New Astron., 15, 433

Motch, C., et al. 2010, A\&A, 523, A92

Muno, M. P., et al. 2003, ApJ, 589, 225

Murray, S. S., et al. 2005, ApJS, 161, 1

Narayan, R., \& McClintock, J. E. 2005, ApJ, 623, 1017

Natalucci, L., Cornelisse, R., Bazzano, A., Cocchi, M., Ubertini, P., Heise, J., in't Zand, J. J. M., \& Kuulkers, E. 1999, ApJ, 523, L45

Nelemans, G., Verbunt, F., Yungelson, L. R., \& Portegies Zwart, S. F. 2000, A\&A, 360, 1011

Nelemans, G., Yungelson, L. R., \& Portegies Zwart, S. F. 2004, MNRAS, 349, 181

Nesterov, V. V., Kuzmin, A. V., Ashimbaeva, N. T., Volchkov, A. A., Röser, S., \& Bastian, U. 1995, A\&AS, 110, 367

Orosz, J. A., \& Hauschildt, P. H. 2000, A\&A, 364, 265

Otero, S. A., Hoogeveen, G. J., \& Wils, P. 2006, IBVS, 5674, 1

Özel, F., Psaltis, D., Narayan, R., \& McClintock, J. E. 2010, ApJ, 725, 1918

Paczynski, B. 1976, in IAU Symp. 73, Structure and Evolution of Close Binary Systems, ed. P. Eggleton, S. Mitton, \& J. Whelan (Cambridge: Cambridge Univ. Press), 75

Patterson, J. 1984, ApJS, 54, 443

Portegies Zwart, S. F., Verbunt, F., \& Ergma, E. 1997, A\&A, 321, 207

Postnov, K. A., \& Yungelson, L. R. 2006, Living Rev. Rel., 9, 6

Predehl, P., \& Schmitt, J. H. M. M. 1995, A\&A, 293, 889

Pretorius, M. L., Knigge, C., O'Donoghue, D., Henry, J. P., Gioia, I. M., \& Mullis, C. R. 2007, MNRAS, 382, 1279

Revnivtsev, M., Sazonov, S., Churazov, E., Forman, W., Vikhlinin, A., \& Sunyaev, R. 2009, Nature, 458, 1142

Rogel, A. B., Cohn, H. N., \& Lugger, P. M. 2008, ApJ, 675, 373

Rogel, A. B., Lugger, P. M., Cohn, H. N., Slavin, S. D., Grindlay, J. E., Zhao, P., \& Hong, J. 2006, ApJS, 163, 160

Romani, R. W. 1992, ApJ, 399, 621

Rucinski, S. M. 1998, AJ, 116, 2998

Sakano, M., Koyama, K., Murakami, H., Maeda, Y., \& Yamauchi, S. 2002, ApJS, 138,19

Scaringi, S., et al. 2010, MNRAS, 401, 2207

Schlegel, D. J., Finkbeiner, D. P., \& Davis, M. 1998, ApJ, 500, 525

Schmitt, J. H. M. M., \& Liefke, C. 2004, A\&A, 417, 651

Sugizaki, M., Mitsuda, K., Kaneda, H., Matsuzaki, K., Yamauchi, S., \& Koyama, K. 2001, ApJS, 134, 77

Taam, R. E., \& Sandquist, E. L. 2000, ARA\&A, 38, 113

Tassoul, J. 1988, ApJ, 324, L71

Torres, C. A. O., Quast, G. R., da Silva, L., de La Reza, R., Melo, C. H. F., \& Sterzik, M. 2006, A\&A, 460, 695

van den Heuvel, E. P. J. 1983, in Accretion-Driven Stellar X-ray Sources, ed. W. H. G. Lewin \& E. P. J. van den Heuvel (Cambridge: Cambridge Univ. Press), 303

van Paradijs, J., \& White, N. 1995, ApJ, 447, L33

Voges, W., et al. 1999, A\&A, 349, 389

Warwick, R. S., Perez-Ramirez, D., \& Byckling, K. 2010, arXiv:1012.1469

Weidenspointner, G., et al. 2008, Nature, 451, 159

Weisskopf, M. C., Brinkman, B., Canizares, C., Garmire, G., Murray, S., \& Van Speybroeck, L. P. 2002, PASP, 114, 1

White, N. E., \& van Paradijs, J. 1996, ApJ, 473, L25

Willems, B., Henninger, M., Levin, T., Ivanova, N., Kalogera, V., McGhee, K., Timmes, F. X., \& Fryer, C. L. 2005, ApJ, 625, 324

Zacharias, N., Urban, S. E., Zacharias, M. I., Wycoff, G. L., Hall, D. M., Monet, D. G., \& Rafferty, T. J. 2004, AJ, 127, 3043 